\title{
INNDICE DE THEIL-T POR ESTRATOS DE RENDA E POR DETERMINANTES DAS DESIGUALDADES DE REMUNERAÇÃO: UMA APLICAÇÃO PARA O MERCADO DE TRABALHO DE SANTA MARIA, RIO GRANDE DO SUL
}

\author{
THEIL-T INDEX BY INCOME STRATUM \\ AND BY DETERMINING THE INCOME INEQUALITY: \\ AN APPLICATION TO THE LABOR MARKET \\ IN SANTA MARIA CITY IN RIO GRANDE DO SUL
}

Data de submissão: 04-12-2012 Aceite: 15-05-2014

Arioane Primon Soares ${ }^{1}$ Luciane Flores Jacobi ${ }^{2}$ Roselaine Ruviaro Zanini ${ }^{3}$ Adriano Mendonça Souza ${ }^{3}$

\section{RESUMO}

Este artigo objetiva apresentar o índice de Theil-T, baseado nas leis da física e na moderna Teoria Matemática da Comunicação de Shannon, para medir a desigualdade de renda. Esse índice permite utilizar dados por estratos de renda, além de estimar as desigualdades inter e intraestratos. Para exemplificar sua aplicação, estimou-se a desigualdade de remuneração no mercado de trabalho formal do município de Santa Maria, Rio Grande do Sul, por características sociais (gênero, faixa etária e grau de instrução) e atributos do próprio mercado (esfera de atuação e atividade econômica) entre 2000 e 2009. Apesar dos índices de desigualdade, dados por essas características, apontarem um nível de desigualdade médio-baixo de acordo com a classificação do Programa das Nações Unidas para o Desenvolvimento (PNUD), ressalta-se a importância de avaliar a distribuição da renda considerando as condições locais de cada microrregião, com a finalidade de direcionar as políticas públicas a corrigirem os determinantes de maior influência nas disparidades.

Palavras-chave: Índice de Theil-T. Desigualdade de renda. Mercado de trabalho.

\footnotetext{
1 Possui graduação em Ciências Econômicas pela Universidade Federal de Santa Maria, UFSM. Santa Maria. Rio Grande do Sul. Brasil. E-mail: arioane@gmail.com

2 Possui graduação em Matemática pela Universidade Federal de Santa Maria, UFSM, mestrado em Engenharia de Produção pela Universidade Federal de Santa Maria, UFSM e doutorado em Agronomia pela Universidade Federal de Santa Maria, UFSM. Atualmente é professor Adjunto da Universidade Federal de Santa Maria. Santa Maria. Rio Grande do Sul. Brasil. E-mail: lucianefj8@gmail.com 3 Possui graduação em Matemática - habilitação em Física pela Faculdade Imaculada Conceição, mestrado em Engenharia de Produção pela Universidade Federal de Santa Maria, UFSM e doutorado em Epidemiologia pela Universidade Federal do Rio Grande do Sul. Atualmente é professor associado I da Universidade Federal de Santa Maria. Santa Maria. Rio Grande do Sul. Brasil. E-mail: rrzanini@terra.com.br 4 Possui graduação em Matemática com Habilitação em Física pela Faculdade de Filosofia Ciências e Letras Imaculada Conceição, FIC, mestrado em Engenharia de Produção Universidade Federal de Santa Maria, UFSM e doutorado em Engenharia de Produção pela Universidade Federal de Santa Catarina, UFSC. Atualmente é Professor Associado do Departamento de Estatística (UFSM) atuando nos curso de Especialização em Estatística e Modelagem Quantitativa (UFSM) e no Mestrado em Engenharia de Produção (UFSM). Santa Maria. Rio Grande do Sul. Brasil. E-mail: amsouza.sm@gmail.com
} 


\begin{abstract}
The main purpose of this is paper is to presents the Theil-T index based on the laws of physics and Shannon's modern mathematical theory of communication to measure income inequality. This index enables to use stratum data by income besides to estimating the inequality intra stratum. The methodology will be illustrated with an application to measure the inequality in the formal labor market in Santa Maria city in Rio Grande do Sul, using social characteristics as gender, age and education level and, attributes of the own market like sphere of activity and economic activity from 2000 to 2009. Although the ex-inequality index calculated using the characteristics above mentioned is classified as medium low according to the PNUD classification, the index emphasizes the importance of assessing the distribution of income considering the local conditions of each micro region in order to direct the public politics to correct the most influential determinants of disparities.
\end{abstract}

\title{
1 INTRODUÇÃO
}

A crescente interligação entre diferentes áreas do conhecimento tem sido fecunda para o surgimento de estudos que teorizam e explicam fenômenos completamente distintos, mas que se mostram guiados por leis naturais e baseados nos mesmos princípios. O índice de Theil-T, tema central deste trabalho, é um exemplo desse tipo de formulação. Sua origem decorre das leis da física que avançaram na explicação matemática acerca do nível informativo de um canal de comunicação, apresentando princípios similares aos que orientam e quantificam a distribuição de renda em uma sociedade.

Apesar de ser pouco conhecido em comparação ao índice de Gini, divulgado pela Organização das Nações Unidas (ONU), por exemplo, o índice de Theil-T é robusto para medir as desigualdades de renda e tem a vantagem de ser uma medida decomponível e adaptável aos dados (THEIL, 1967 apud FROSINI, 2012; RAMOS, 2007). Usualmente, a análise da desigualdade de renda exige medidas de rendas individuais, o que, em muitos casos, inviabiliza estudos sobre o tema. Porém, o índice de Theil-T admite dados por estratos ou faixas de renda e avalia a desigualdade tanto dentro de cada estrato quanto entre os estratos (HOFFMANN, 1979; FUNDAÇÃO JOÃO PINHEIRO, 1998; FROSINI, 2012).

Assim, este artigo objetiva introduzir a medida de desigualdade de Theil-T para analisar dados por estrato de renda e mostrar sua aplicação por meio de um estudo de caso referente à distribuição de renda no mercado de trabalho formal do município de Santa Maria, Rio Grande do Sul, no período de 2000 a 2009. Para isso, consideram-se como prováveis determinantes para as desigualdades fatores que configuram o mercado de trabalho (natureza dos estabelecimentos e atividades econômicas) e características sociais (gênero, idade e grau de instrução). Com essa abordagem, pretende-se mostrar a importância do monitoramento das desigualdades de renda sob uma perspectiva microrregional, delimitada espacialmente, em que se vislumbram as oportunidades ocupacionais, o sistema educacional, as atividades econômicas e o próprio grau de desenvolvimento das atividades.

\section{O ÍNDICE DE THEIL-T}

Conforme Matos (2005), a medida de desigualdade de renda de Theil-T teve sua origem com os conceitos da teoria da informação. Essa teoria, baseada na mensuração do conteúdo informativo de uma mensagem, é um ramo da teoria das probabilidades que lida com sistemas de comunicação. Através das probabilidades a priori (sem informação) e a posteriori (com infor- 
mação), pode-se avaliar o conteúdo informativo da mensagem recebida. De modo geral, quanto maior for a diferença entre as probabilidades a posteriori e a priori, maior será o conteúdo informativo da informação, ao passo que a igualdade ou uma pequena flutuação entre essas probabilidades evidencia que a mensagem teve nenhum ou pouco conteúdo informativo (MATOS, 2005).

\subsection{Elementos da teoria da informação}

Para Shannon (1948), o valor informativo que se atribui a uma mensagem está associado a uma probabilidade de ocorrência do evento $x$ noticiado. Ou seja, considerando-se uma notícia jornalística, em casos extremos, quando a probabilidade de ocorrência é certa $(p(x)=1)$, a mensagem de ocorrência do evento não tem nenhum valor informativo. Mas, quando a probabilidade de ocorrência é muito baixa $(p(x)$ tende a 0$)$, o valor informativo da mensagem pode chegar ao infinito, como é o caso de uma notícia recebida com muita surpresa. Dessa forma, de acordo com Shannon (1948), quanto menor for o valor de $p(x)$, maior será o conteúdo informativo da mensagem, de modo que o conteúdo informativo da mensagem que afirma que determinado evento "ocorreu" é dado por:

$$
h(x)=\mathrm{h} \frac{1}{p(x)}=-\mathrm{h} p(x)
$$

A respeito dessa equação, de acordo com Hoffmann (1998), torna-se útil esclarecer duas peculiaridades. A primeira é que a unidade de medida do conteúdo informativo de uma mensagem, em teoria da informação, é dada em bits (binary units), quando se utilizam logaritmos de base 2, ou em nits (natural units), quando se empregam logaritmos naturais. Essas unidades de medidas são intercambiáveis, de forma que 1 bit equivale a 0,693 nit e que 1 nit é igual a 1,443 bit. No entanto, neste estudo, devido ao seu uso ser mais frequente, emprega-se o nit, dispensando-se, assim, a alusão ao bit na sequência deste trabalho. A segunda particularidade refere-se à escolha da função logarítmica, pelo fato de esta permitir a adição de eventos independentes. Assim, sendo $A 1$ e $A 2$ eventos disjuntos com probabilidades de ocorrência $p(x 1)$ e $p(x 2)$, o conteúdo informativo da mensagem de ocorrência desses dois eventos é dado pela soma do valor informativo das mensagens de que $A 1$ e $A 2$ ocorreram, conforme a seguinte equação descrita por Shannon (1948):

$$
h\left(x_{1} x_{2}\right)=2 \mathrm{~h} \frac{1}{p\left(x_{1}\right) p\left(x_{2}\right)}=\mathrm{h} \frac{1}{p\left(x_{1}\right)}+\mathrm{h} \frac{1}{p\left(x_{2}\right)}=h\left(x_{1}\right)+h\left(x_{2}\right)
$$

Já para medir o valor informativo de uma previsão, de uma mensagem incerta ou de uma mensagem sujeita a erro, como no caso da informação de que um fenômeno natural (chuva, furacão, abalo sísmico etc.) irá incidir, recorre-se, conforme Hoffmann (1998), às probabilidades a priori $p(x)$ (probabilidade de o evento ocorrer antes de ser recebida a mensagem) e a posteriori $p(y)$ (probabilidade de o evento ocorrer depois de recebida a mensagem). Matematicamente:

$$
h=\mathrm{h} \frac{p(y)}{p(x)}
$$

Pode-se observar que a equação (1) é um caso particular da (3), em que $p(y)$ é igual à unidade. Assim, segundo Hoffmann (2006), para um universo de $n$ possíveis eventos $A i(i=1, \ldots, n)$ mutuamente exclusivos, com probabilidades $p(x i)$ de ocorrência, o somatório das probabilidades é igual a 1, ou seja:

$$
\sum_{i=1}^{n} p\left(x_{i}\right)=1
$$


A entropia é dada pela esperança matemática da mensagem informativa de que determinado evento (Ai) "ocorreu" (MATOS, 2005).

$$
H(x)=E\left[h\left(p\left(x_{i}\right)\right)\right]=\sum_{i=1}^{n} p\left(x_{i}\right) h\left(x_{i}\right)=\sum_{i=1}^{n} p\left(x_{i}\right) \mathrm{h} \frac{1}{p\left(x_{i}\right)}=-\sum_{i=1}^{n} p\left(x_{i}\right) \mathrm{h} p\left(x_{i}\right)
$$

Segundo Hoffmann (2006), quando $p\left(x_{i}\right)=0$, adota-se a notação especial de que $p\left(x_{i}\right)$ h $p\left(x_{i}\right)=0$, dado que: $\lim _{p(x) \rightarrow 0}[p(x) \mathrm{h} p(x \rrbracket=0$

Para valores de $p\left(x_{i}\right)=0$, tais que $0<p\left(x_{i}\right) \leq 1$, tem-se que:

$$
\begin{aligned}
& H(x)=\sum_{i=1}^{n} p\left(x_{i}\right) \mathrm{h} \frac{1}{p\left(x_{i}\right)}=-\sum_{i=1}^{n} p\left(x_{i}\right) \mathrm{h} p\left(x_{i}\right) \geq 0 \\
& \text { Pois: } \frac{1}{p\left(x_{i}\right)} \geq 1 \text { e } \mathrm{h} \frac{1}{p\left(x_{i}\right)} \geq 0
\end{aligned}
$$

Portanto, quando uma das probabilidades for igual a $1 \mathrm{e}$, por conseguinte, as demais forem iguais a $0, H(x)=0$. Contudo, para determinar o valor máximo da entropia sujeita à restrição de que $\Sigma p\left(x_{i}\right)=1$, utiliza-se o método do multiplicador de Lagrange, conforme a seguinte equação:

$$
-\Sigma p(x)_{i} \mathrm{~h} p\left(x_{i}\right)-\mathrm{I}\left[\Sigma p\left(x_{i}\right)-1\right]
$$

Igualando-se a zero as derivadas parciais da função em relação à $x i$, obtém-se que: h $p\left(x_{i}\right)=-(1+1)$, para $i=1, \ldots, n$.

Por isso, o máximo de $H(x)$, que se configura na "desordem" mais elevada de um sistema, ocorre quando todas as probabilidades são idênticas entre si ou igualmente prováveis, isto é, iguais a $1 / n$. Dessa forma:

$$
\begin{aligned}
& H(x)=\Sigma p\left(x_{i}\right) \mathrm{h} \frac{1}{p\left(x_{i}\right)}=\Sigma \frac{1}{n} \mathrm{~h} n=\mathrm{h} n \\
& \text { Logo: } \\
& 0 \leq H(x) \leq \mathrm{h} n
\end{aligned}
$$

Para Matos (2005), é possível calcular o valor informativo de uma mensagem incerta quando, após o recebimento de uma mensagem (uma previsão) qualquer, as probabilidades $a$ priori se alterem devido à mensagem, mas sem que ocorra, necessariamente, determinado evento com probabilidade igual a 1 (evento certo).

Segundo Hoffmann (1998), considerando-se o universo de $n$ prováveis eventos $A i$, mutuamente exclusivos, associados às probabilidades de ocorrência $x i(i=1, \ldots, n)$, e, sendo as probabilidades a priori $p(x i)$ transformadas em probabilidades a posteriori $p(y i)$ (probabilidade de ocorrência do evento após o recebimento da mensagem), a informação esperada de uma mensagem incerta $(U)$ é dada por:

$$
U(y: x)=\sum_{i=1}^{n} p\left(y_{i}\right) \mathrm{h} \frac{p\left(y_{i}\right)}{p\left(x_{i}\right)}
$$


A determinação do conteúdo informativo de uma mensagem certa segundo a equação (1) é um caso particular de (9), em que uma probabilidade a posteriori é igual a 1 e em que as outras probabilidades são, consequentemente, nulas.

\subsection{Relação da entropia com o índice Theil-T}

Considerando uma população de $n$ pessoas, em que cada uma recebe uma fração não negativa ( $y i \geq 0, \operatorname{com} i=1, \ldots, n)$ da renda total, com base na equação (4), em que $p(y i)$ tem as mesmas propriedades probabilísticas pertinentes a um universo de eventos, segundo Matos (2005), define-se a entropia da distribuição de renda $H(y)$ como:

$$
H(y)=\sum_{i=1}^{n} p\left(y_{i}\right) \mathrm{h} \frac{1}{p\left(y_{i}\right)}
$$

Conforme a expressão (8), pode-se afirmar que: $0<H(y) \leq \mathrm{h} n$

Quando $p(y i)=1 / n$, para todo $i=1, \ldots, n$, a entropia da distribuição de renda $H(y)=\ln$ $n$, ou seja, há perfeita igualdade na distribuição de renda. Entretanto, quando $p(y j)=1$ e $p(x i)=$ 0 , para todo $i \neq j, H(y)=0$, o que aponta para um caso de perfeita desigualdade na distribuição.

Assim, a entropia de Theil é uma medida do grau de igualdade da distribuição da renda. No entanto, conforme Hoffmann (2006), Theil achou mais interessante transformar a entropia da renda em uma medida de desigualdade dessa distribuição. Para isso, subtraiu a entropia de seu próprio valor máximo, obtendo o índice de Theil-T:

$$
T=\mathbf{h} n-H(x)=\sum_{i=1}^{n} y_{i}{\mathrm{~h}{ }_{i}}_{i}=\sum_{i=1}^{n} y_{i} \mathrm{~h} \frac{y_{i}}{\frac{1}{n}}
$$

Em que: yi é a fração da renda apropriada por pessoa, e $1 / n$ é a fração da população correspondente a cada indivíduo.

Para o cálculo da equação (11), é preciso dispor das rendas individuais da população, fato que pode dificultar muito o processo de estimação (FROSINI, 2012). Hoffmann (1979) deixa isso bem claro ao afirmar que

Os dados disponíveis para o cálculo de medidas de concentração da distribuição de renda ou da riqueza em uma população consistem, frequentemente, no número de pessoas e nos totais de renda ou riqueza referentes a um certo número de estratos, e não dispomos (ou são de difícil acesso) das informações referentes à renda ou riqueza de cada indivíduo. É comum, nesses casos, calcular-se a medida de concentração referente à desigualdade entre estratos, desprezando-se a desigualdade dentro destes. Esse procedimento pode levar a uma séria subestimação do grau de desigualdade real (p.720).

Tendo isso em vista, Hoffmann (1979) e Ramos (1990) descrevem metodologias de cálculo adaptadas para as medidas de desigualdade da renda de Theil. Dentre elas, está a que Hoffmann (1979) preferiu tratar como a redundância $(R)$ de uma distribuição de renda ao invés de índice de Theil-T. A diferença entre estas medidas é dada pela seguinte equação:

$$
T=1-e^{-R}
$$

Ou seja, o índice de Theil-T (ou Dual de uma distribuição) consiste na unidade subtraída do inverso da função exponencial da redundância. Nessa circunstância, ele é comparável ao índice de Gini, pois passa a variar entre 0 e 1 . Logo:

$$
0 \leq T \leq 1
$$




\section{3 Índice Theil-T ou redundância de uma distribuição para dados por estratos de renda}

Conforme Matos (2005), Ramos e Vieira (2001) e Frosini (2012), as medidas de desigualdade de Theil possuem uma importante vantagem em relação a outros índices de desigualdade, pois podem ser decompostas. Quando é possível e interessante agrupar os indivíduos em grupos disjuntos e avaliar as rendas individuais, pode-se decompor o valor da medida total em variação intergrupos e variação intragrupos.

$\mathrm{Na}$ indisponibilidade das rendas individuais de uma população e supondo a renda de uma população $(\mathrm{Y})$ computada de acordo com $k$ faixas ou estratos de renda, considera-se $n h$ a contagem de indivíduos em cada faixa de renda e yh $(h=1, \ldots, k)$ a renda total dos indivíduos que se situam na $h$-ésima faixa de renda. Nesse contexto, a população é dada por: $N=\sum_{h=1}^{k} n_{h}$

A renda média de cada estrato é: $\mathrm{m}_{h}=\frac{y_{h}}{\sum_{k} n_{h}}$

E a renda média da população é: $\mathrm{m}=\frac{\sum_{h=1}^{k^{n_{h}}} \mathrm{~m}_{h} n_{h}}{N}$

A proporção da população que se situa na $h$-ésima faixa de renda é: $\mathrm{p}_{h}=\frac{n_{h}}{N}$

E a respectiva proporção da renda total é: $I_{h}=\frac{y_{h}}{Y}$

Logo, a redundância da distribuição da renda é dada por:

$R=R_{e}+\sum_{h=1}^{k} I_{h} R_{h}$

$R e$ é a redundância ou o índice de Theil-T entre estratos de renda, e $R h$ é a redundância ou o índice de Theil-T dentro dos estratos. Os índices $R e$ e $R h$ são calculados, respectivamente, pelas seguintes equações:

$$
\begin{gathered}
R_{e}=\sum_{h=1}^{k} I_{h} \mathrm{~h} \frac{I_{h}}{\mathrm{p}_{h}} \\
R_{h}=\frac{a^{2}}{2 \mathrm{q}_{h}}\left[1-\left(\frac{2 a}{\mathrm{q}}+3\right)<-1\right] \frac{b}{a}+\mathrm{h} \frac{b}{\mathrm{~m}_{h}}+\frac{1}{\mathrm{q}_{h}}<-1\left(a \mathrm{q}+\frac{\mathrm{q}^{2}}{\mathrm{l}}\right)-\frac{1}{2 \mathrm{~m}_{h}}\left(a+\frac{\mathrm{q}}{2}\right)
\end{gathered}
$$

$R e$ e $R h$ avaliam, respectivamente, a desigualdade entre os estratos de renda e dentro de cada estrato de renda.

Segundo Hoffmann (1979), como $R h$ é de difícil estimação, tende-se a utilizar $R=R e$. No entanto, essa medida da desigualdade é adequada somente se houver perfeita igualdade de renda dentro dos estratos. Caso contrário, usando-se apenas $R e$ para estimar $R$, incorre-se na subestimação do verdadeiro grau de desigualdade.

Nessa conjuntura, Hoffmann (1979) apresenta métodos para a estimação de Rh. O que se utiliza neste estudo é baseado em dois pressupostos:

a) a distribuição de renda dentro de estratos com limites finitos segue uma distribuição linear;

b) dentro de estratos de rendas mais elevadas, quando o estrato é aberto à direita ou delimitado pelo símbolo de infinito $(\infty)$, admite-se que a distribuição é a de Pareto com dois parâmetros. 
Hoffmann (1979) ressalta o fato de não se pretender afirmar que a distribuição real tenha função de densidade linear, mas que essa distribuição é usada como forma de estimar a desigualdade de renda dentro das faixas e, por isso, pode superestimar ou subestimar a desigualdade. Contudo, afirma que o método é melhor que, simplesmente, ignorar ou considerar que a desigualdade dentro de uma faixa finita de renda é uniforme.

Assim, supondo-se um estrato com limites finitos $E_{h-1}$ (limite inferior do estrato) e $E_{h}$ (limite superior do estrato) e cuja remuneração média seja $\mu_{\mathrm{h}}$, os parâmetros aplicados na equação (16), para o cálculo da desigualdade dentro dos estratos finitos, seguem três casos, conforme mostra a Tabela 1, exposta a seguir.

Tabela 1 - Parâmetros específicos utilizados no cálculo de $R h$

\begin{tabular}{ccccc}
\hline Se $\mu_{h}-E_{h} / E_{h}-E_{h-1}$ & $\lambda$ & $a$ & $b$ & $\theta$ \\
\hline Menor que $1 / 3$ & $1 / 3$ & $E_{h-1}$ & $3 \mu_{h}-2 E_{h-1}$ & $3\left(\mu_{h}-E_{h-1}\right)$ \\
Entre $1 / 3$ e $2 / 3$ & $1 / 2$ & $E_{h-1}$ & $E_{h}$ & $E_{h}-E_{h-1}$ \\
Maior que 2/3 & $2 / 3$ & $3 \mu_{h}-2 E_{h}$ & $E_{h}$ & $3\left(E_{h}-\mu_{h}\right)$ \\
\hline
\end{tabular}

Fonte: baseado em Hoffmann (1979).

Eh-1 - Limite inferior do estrato; Eh - Limite superior do estrato;

Para o estrato com limite infinito, pressupõe-se que a distribuição seja a de Pareto com dois parâmetros, de modo que o cálculo de desigualdade dentro do estrato $(R h)$ é dado por:

$$
R_{h}=\frac{\mathrm{m}_{h}}{E_{h-1}}-1-\mathrm{h} \frac{\mathrm{m}_{h}}{E_{h-1}}
$$

Onde $E_{h-1}=$ limite inferior do estrato de renda e $\mu_{h}=$ remuneração média dos trabalhadores por estrato $(h)$.

\section{MATERIAIS E MÉTODOS}

Esta é uma pesquisa de caráter exploratório. De acordo com Gil (2002 apud LENGLER, 2008), uma pesquisa exploratória envolve levantamento bibliográfico, entrevistas com pessoas que tiveram experiência práticas com o problema pesquisado e análise de exemplos que estimulem a compreensão da situação abordada. Além disso, esta pesquisa, segundo Baisch et al. (2012), caracteriza-se como descritiva, pois objetiva a descrição das características da desigualdade de renda no mercado.

Para realizar este estudo, foram utilizadas informações obtidas a partir da Relação Anual de Informações Sociais (RAIS) do Ministério do Trabalho e Emprego (MTE) que abrangem apenas o setor formal do mercado de trabalho de Santa Maria, Rio Grande do Sul, no período de 2000 a 2009. As análises foram realizadas por meio do índice de Theil-T, estratificado por gênero, faixa etária, grau de instrução, esfera de atuação e atividade econômica.

Conforme Ramos e Vieira (2001), estudar as desigualdades no mercado de trabalho tem algumas peculiaridades, pois o mercado apresenta fontes de dispersão dos salários intrínsecas ao seu funcionamento e outras que provêm de desigualdades sociais preexistentes. Assim, o mercado pode funcionar como revelador das desigualdades, ao remunerar distintamente os trabalhadores para compensar diferenças não pecuniárias dos postos de trabalho como periculosidade e insalubridade ou ao remunerar de acordo com dotações desiguais de qualificações (educação e experiência), bem como pode atuar como gerador das desigualdades, ao remunerar diferente- 
mente indivíduos que possuem potenciais produtivos e colocações similares sem base tangível ou baseado em fatores contraproducentes como gênero e raça.

Os parâmetros e as variáveis utilizadas para estimar a medida de desigualdade de Theil -T, bem como suas definições, consideram as características do mercado de trabalho evidenciadas por Ramos e Vieira (2001) e são apresentadas a seguir no Quadro 1.

\begin{tabular}{|c|c|}
\hline$N$ - Estoque de emprego & $\begin{array}{l}\text { Número médio de trabalhadores com vínculo empregatício até } 31 \text { de dezem- } \\
\text { bro de cada ano }\end{array}$ \\
\hline$Y$ - Massa salarial & $\begin{array}{l}\text { Produto entre o estoque de emprego e a remuneração média salarial dos } \\
\text { trabalhadores no ano considerado }\end{array}$ \\
\hline SM - Salário(s) mínimo(s) & Unidade de medida da renda recebida pelos trabalhadores \\
\hline$h$ - Estratos de renda & $\begin{array}{l}\text { Faixas de renda em salários mínimos em que ficam distribuídos os trabalha- } \\
\text { dores de acordo com suas remunerações médias anuais }\end{array}$ \\
\hline $\begin{array}{l}\text { Faixas de renda }(h=12) \\
\text { dos trabalhadores do } \\
\text { mercado de trabalho } \\
\text { formal }\end{array}$ & $\begin{array}{l}\text { Até meio salário mínimo } \\
\text { De 0,51 a 1,00 salário mínimo } \\
\text { De 1,01 a 1,50 salário mínimo } \\
\text { De 1,51 a 2,00 salários mínimos } \\
\text { De 2,01 a 3,00 salários mínimos } \\
\text { De 3,01 a 4,00 salários mínimos } \\
\text { De 4,01 a 5,00 salários mínimos } \\
\text { De 5,01 a 7,00 salários mínimos } \\
\text { De 7,01 a } 10,00 \text { salários mínimos } \\
\text { De 10,01 a } 15,00 \text { salários mínimos } \\
\text { De 15,01 a } 20,00 \text { salários mínimos } \\
\text { Mais de } 20 \text { salários mínimos }\end{array}$ \\
\hline Grau de instrução & $\begin{array}{l}\text { Até } 5^{a} \text { série do Ensino Fundamental (Até 5aㅡ) } \\
5 \text { a série ao Ensino Fundamental Completo (5a - EFC) } \\
\text { Ensino Médio Incompleto ao Ensino Médio Completo (EMI - EMC) } \\
\text { Acima do Ensino Médio Completo (Acima EMC inclui ensino superior incom- } \\
\text { pleto, completo, mestres e doutores) }\end{array}$ \\
\hline Faixa etária & $\begin{array}{l}10 \text { - } 24 \text { (de } 10 \text { a } 24 \text { anos) } \\
25 \text { - } 29 \text { (de } 25 \text { a } 29 \text { anos) } \\
30 \text { - } 39 \text { (de } 30 \text { a } 39 \text { anos) } \\
40 \text { - } 49 \text { (de } 40 \text { a } 49 \text { anos) } \\
50-64 \text { (de } 50 \text { a } 64 \text { anos) } \\
65 \text { ou + (Mais de } 65 \text { anos) }\end{array}$ \\
\hline Gênero & $\begin{array}{l}\text { M-Masculino } \\
\text { F- Feminino }\end{array}$ \\
\hline Esfera de atuação & $\begin{array}{l}\text { PUB - Setor Público: abrange os setores públicos federal, estadual e munici- } \\
\text { pal, além de entidades empresariais estatais } \\
\text { PRI - Setor Privado: composto de entidades empresariais privadas, sem fins } \\
\text { lucrativos, pessoas físicas e outras formas de organização legal }\end{array}$ \\
\hline Atividade econômica & $\begin{array}{l}\text { IND - Indústria } \\
\text { CCI - Construção civil } \\
\text { COM - Comércio } \\
\text { SRV - Serviços } \\
\text { AGR - Agropecuária }\end{array}$ \\
\hline
\end{tabular}

Quadro 1 - Parâmetros e variáveis: suas definições e subdivisões 
Cabe observar que, para fins de cálculo, subtraiu-se do total de trabalhadores o número de trabalhadores com renda ignorada, de acordo com os dados da RAIS/MTE. Os cálculos foram efetuados utilizando-se a Planilha Excel, uma vez que não existem pacotes estatísticos que estimem o índice de Theil-T.

A classificação das desigualdades de renda seguiu a orientação de Santos (2007), que observa que o Programa das Nações Unidas para o Desenvolvimento (PNUD) leva em conta cinco categorias de desigualdade: baixa $(0,000-0,2999)$; média-baixa $(0,300-0,449)$; média $(0,450-$ 0,599); média-alta (0,600-0,749); e alta (0,750-1,000).

Com o objetivo de ilustrar a aplicação desta técnica, foi calculado o índice Theil-T para verificar a desigualdade entre gêneros no mercado de trabalho formal de Santa Maria. A Tabela 2, exposta a seguir, apresenta os dados acerca da renda média e do número de trabalhadores para o gênero masculino no ano 2000.

A massa salarial $\left(\mathrm{y}_{\mathrm{h}}\right)$ do estrato 1 representa a renda total média dos 80 trabalhadores que recebem até meio salário mínimo. Para determinar $\mu_{\mathrm{h}}$ que representa a renda média de cada trabalhador do estrato, basta dividir $y_{h}$ por $n_{h}\left(\mu_{h}=0,47\right)$. Para calcular o índice de Theil-T dentro dos estratos, é necessário utilizar a equação 16, que depende de parâmetros específicos. Esses parâmetros estão compilados, passo a passo, na Tabela 1.

Tabela 2 - Renda média $\left(\mathrm{y}_{\mathrm{h}}\right)$, número de trabalhadores $\left(\mathrm{n}_{\mathrm{h}}\right)$ do gênero masculino, número do estrato (h), faixa de renda, limite inferior do estrato $\left(E_{h-1}\right)$ e limite superior do estrato $\left(E_{h}\right)$

\begin{tabular}{cccccccccc}
\hline $\mathrm{h}$ & Faixas de renda & $\mathrm{n}_{\mathrm{h}}$ & $\mathrm{V}_{\mathrm{h}}$ & $\mathrm{E}_{\mathrm{h}-1}$ & $\mathrm{E}_{\mathrm{h}}$ & $\mu_{\mathrm{h}}$ & $\frac{\left(\mathrm{m}_{h}-E_{h}\right)}{E_{h}-E_{h-1}}$ & $\mathrm{R}_{\mathrm{h}}$ & $\mathrm{V}_{\mathrm{h}}^{*} \mathrm{R}_{\mathrm{h}}$ \\
\hline 1 & ATÉ 0,50 SM & 80 & 37,38 & 0,00 & 0,50 & 0,47 & 0,93 & 0,001 & 0,000 \\
2 & $0,51-1,00 \mathrm{SM}$ & 572 & 439,33 & 0,51 & 1,00 & 0,77 & 0,53 & 0,005 & 0,000 \\
3 & $1,01-1,50 \mathrm{SM}$ & 1424 & 1826,33 & 1,01 & 1,50 & 1,28 & 0,56 & $-0,012$ & 0,000 \\
4 & $1,51-2,00 \mathrm{SM}$ & 4383 & 7699,55 & 1,51 & 2,00 & 1,76 & 0,50 & 0,002 & 0,000 \\
5 & $2,01-3,00 \mathrm{SM}$ & 5513 & 13391,85 & 2,01 & 3,00 & 2,43 & 0,42 & 0,032 & 0,004 \\
6 & $3,01-4,00 \mathrm{SM}$ & 2739 & 9449,04 & 3,01 & 4,00 & 3,45 & 0,44 & 0,017 & 0,002 \\
7 & $4,01-5,00 \mathrm{SM}$ & 1527 & 6802,74 & 4,01 & 5,00 & 4,45 & 0,45 & 0,012 & 0,001 \\
8 & $5,01-7,00 \mathrm{SM}$ & 2229 & 13169,66 & 5,01 & 7,00 & 5,91 & 0,45 & 0,018 & 0,002 \\
9 & $7,01-10,00 \mathrm{SM}$ & 1259 & 10349,99 & 7,01 & 10,00 & 8,22 & 0,40 & 0,034 & 0,003 \\
10 & $10,01-15,00 \mathrm{SM}$ & 744 & 9040,05 & 10,01 & 15,00 & 12,15 & 0,43 & 0,030 & 0,003 \\
11 & $15,01-20,00 \mathrm{SM}$ & 527 & 9239,82 & 15,01 & 20,00 & 17,53 & 0,51 & 0,002 & 0,000 \\
12 & MAIS DE 20,0 SM & 803 & 24786,16 & 20,01 & $\infty$ & 30,87 & - & 0,109 & 0,025 \\
\hline Total & & 21800 & 106231,9 & & & 4,87 & & & 0,040 \\
\hline
\end{tabular}

Assim, para a primeira faixa de renda, tem-se que a relação $\left(\mu_{h}-E_{h-1}\right) /\left(E_{h}-E_{h-1}\right)$ é maior que 2/3; portanto, assume-se que $\lambda=2 / 3, a=\left(3 \mu_{h}-2 E_{h}\right)=(3.0,47-2.0,5)=0,4, b=E_{h}=0,5$ e $\theta$ $=3\left(E_{h}-\mu_{h}\right)=3(0,5-0,47)=0,10$. Substituindo esses valores na equação 16 , obtém-se que $R_{h} e ́$ 0,001 para a primeira faixa de renda.

Para as demais faixas de renda da Tabela 2, a relação $\left(\mu_{h}-E_{h-1}\right) /\left(E_{h}-E_{h-1}\right)$ ficou entre $1 / 3$ e 2/3; assim, os valores dos parâmetros ficam estabelecidos da seguinte forma: $\lambda=1 / 2, a=$ $E_{h-1}, b=E_{h}$ e $\theta=E_{h}-E_{h-1}$. Para o último estrato, onde não se tem o limite superior definido, o $R_{h}$ foi calculado utilizando-se a equação 17.

Para calcular o índice de Theil-T, é necessário também determinar o valor da desigualdade entre os estratos de renda $R_{e}$. Essa desigualdade é calculada por meio da equação 15 . Após a determinação de $R_{e^{\prime}}$ utilizando a equação 14 , é possível determinar o valor da redundância e, 
por meio da equação 12 , o valor de Theil-T, que, para o gênero masculino no ano de 2000 , foi de 0,397. Todos os demais índices apresentados na Tabela 3 foram calculados dessa forma.

\section{ESTUDO DE CASO: DESIGUALDADE DE RENDA NO MERCADO DE TRABALHO DE SANTA MARIA DE 2000 A 2009}

O município de Santa Maria, Rio Grande do Sul, tem apresentado incrementos em sua renda per capita ao longo dos últimos anos, sendo considerado, conforme Denardin et al. (2012), basicamente agrícola do ponto de vista econômico, formado por pequenas e médias propriedades e voltado para a atividade comercial. Segundo dados divulgados em 2008 pela Fundação de Economia e Estatística (FEE) do estado do Rio Grande do Sul, o Produto Interno Bruto (PIB) de Santa Maria apresentou, somente no período de 2000 a 2007, um crescimento de mais de $122 \%$, enquanto que a população do município passou de 243.611 em 2000 para 270.363 habitantes em 2009, um aumento aproximado de $11 \%$ para a década. Ou seja, o crescimento da renda foi superior ao crescimento populacional; porém, sabe-se que a renda per capita não é um bom indicador para o fator distributivo.

Contudo, a distribuição de renda em uma sociedade constitui um indicador de bem-estar social (FIGUEIREDO; ZIELGMANN, 2009, p. 7). Um nível de desigualdade muito elevado pode ter consequências ameaçadoras para ricos e pobres, pois inviabiliza oportunidades semelhantes de inclusão social, incitando o aumento da violência. De acordo com Bêrni, Marquetti e Kloeckner (2002, p. 445), a desigualdade distributiva e a exclusão podem corromper várias dimensões da vida dos cidadãos e espalhar "crime, desnutrição, doença, ignorância e injustiça [...] de forma enviesada entre pobres e ricos".

No que se refere às desigualdades do mercado de trabalho de Santa Maria, embora a teoria do desenvolvimento regional situe o município no grupo dos menos desenvolvidos do estado (AREND, 2005), os índices de Theil, de acordo com a classificação do PNUD, apontam para uma desigualdade médio-baixa nos rendimentos dos trabalhadores do seu mercado de trabalho. Os índices de desigualdade encontrados são maiores nas variáveis faixa etária e gênero (Tabela 3) para o período de 2000 a 2009. As categorias 65 anos ou mais e 50 a 64 anos apresentaram, respectivamente, coeficientes médio-baixos de desigualdade de 0,423 e 0,375 , conforme a classificação do PNUD. Logo, essa desigualdade fica aquém da realidade brasileira - índice de Theil = 0,602, em 2006, conforme Araujo, Salvato e Souza (2008) -, mas superior à desigualdade de muitos países que podem ser considerados desenvolvidos. 
ÍNDICE DE THEIL-T POR ESTRATOS DE RENDA E POR DETERMINANTES DAS DESIGUALDADES DE REMUNERAÇÃO: UMA APLICAÇÃO PARA O MERCADO DE TRABALHO DE SANTA MARIA, RIO GRANDE DO SUL

Tabela 3 - Índice de Theil-T por gênero, faixa etária, grau de instrução, esfera de atuação e atividade econômica para o mercado de trabalho formal de Santa Maria (2000-2009)

\begin{tabular}{|c|c|c|c|c|c|c|c|c|c|c|c|}
\hline & 2000 & 2001 & 2002 & 2003 & 2004 & 2005 & 2006 & 2007 & 2008 & 2009 & Média \\
\hline \multicolumn{12}{|l|}{ Gênero } \\
\hline$M$ & 0,397 & 0,386 & 0,383 & 0,364 & 0,362 & 0,356 & 0,353 & 0,343 & 0,346 & 0,357 & 0,365 \\
\hline $\mathrm{F}$ & 0,335 & 0,338 & 0,335 & 0,320 & 0,323 & 0,324 & 0,324 & 0,321 & 0,330 & 0,343 & 0,329 \\
\hline \multicolumn{12}{|l|}{ Faixa Etária } \\
\hline $10-24$ & 0,176 & 0,159 & 0,141 & 0,124 & 0,128 & 0,115 & 0,103 & 0,107 & 0,109 & 0,113 & 0,127 \\
\hline $25-29$ & 0,211 & 0,234 & 0,229 & 0,225 & 0,224 & 0,221 & 0,217 & 0,196 & 0,203 & 0,208 & 0,217 \\
\hline $30-39$ & 0,301 & 0,313 & 0,311 & 0,301 & 0,296 & 0,292 & 0,300 & 0,286 & 0,290 & 0,302 & 0,299 \\
\hline $40-49$ & 0,366 & 0,363 & 0,347 & 0,328 & 0,321 & 0,315 & 0,319 & 0,319 & 0,330 & 0,337 & 0,334 \\
\hline $50-64$ & 0,412 & 0,410 & 0,402 & 0,382 & 0,380 & 0,372 & 0,351 & 0,341 & 0,345 & 0,358 & 0,375 \\
\hline 65 ou + & 0,465 & 0,462 & 0,438 & 0,455 & 0,419 & 0,416 & 0,397 & 0,382 & 0,395 & 0,398 & 0,423 \\
\hline \multicolumn{12}{|c|}{ Grau de instrução } \\
\hline Até $5^{a}$ & 0,181 & 0,174 & 0,200 & 0,143 & 0,159 & 0,146 & 0,150 & 0,164 & 0,160 & 0,138 & 0,162 \\
\hline $5^{a}-E F C$ & 0,185 & 0,171 & 0,175 & 0,164 & 0,172 & 0,168 & 0,168 & 0,167 & 0,164 & 0,169 & 0,170 \\
\hline EMI - EMC & 0,291 & 0,282 & 0,274 & 0,253 & 0,247 & 0,246 & 0,240 & 0,223 & 0,227 & 0,225 & 0,251 \\
\hline Acima EMC & 0,322 & 0,318 & 0,307 & 0,295 & 0,295 & 0,293 & 0,296 & 0,291 & 0,301 & 0,303 & 0,302 \\
\hline \multicolumn{12}{|c|}{ Esfera de atuação } \\
\hline PUB & 0,284 & 0,266 & 0,254 & 0,284 & 0,243 & 0,228 & 0,225 & 0,218 & 0,224 & 0,217 & 0,244 \\
\hline PRI & 0,324 & 0,305 & 0,249 & 0,324 & 0,258 & 0,254 & 0,239 & 0,241 & 0,232 & 0,228 & 0,265 \\
\hline \multicolumn{12}{|c|}{ Atividade Econômica } \\
\hline IND & 0,300 & 0,238 & 0,292 & 0,291 & 0,300 & 0,293 & 0,267 & 0,281 & 0,262 & 0,246 & 0,277 \\
\hline $\mathrm{CCl}$ & 0,175 & 0,152 & 0,144 & 0,093 & 0,084 & 0,094 & 0,101 & 0,107 & 0,127 & 0,116 & 0,119 \\
\hline COM & 0,230 & 0,225 & 0,214 & 0,210 & 0,200 & 0,179 & 0,163 & 0,166 & 0,164 & 0,173 & 0,192 \\
\hline SRV & 0,370 & 0,364 & 0,359 & 0,327 & 0,325 & 0,322 & 0,334 & 0,327 & 0,337 & 0,348 & 0,341 \\
\hline AGR & 0,111 & 0,109 & 0,090 & 0,042 & 0,055 & 0,041 & 0,044 & 0,055 & 0,060 & 0,074 & 0,068 \\
\hline
\end{tabular}

Além disso, nota-se pela Tabela 3 que as desigualdades se acentuam com o aumento da idade e do grau de instrução, sugerindo a existência de correlação entre os níveis de desigualdade dessas duas variáveis. A variável idade, por si só, parece não esclarecer o mais alto coeficiente de iniquidade entre os trabalhadores de Santa Maria, mas o grau de instrução e a experiência, intimamente interligados a essa variável, sugerem uma explicação razoável.

A categoria Acima EMC (Acima Ensino Médio Completo = nível superior incompleto, completo, mestres, doutores), da variável grau de instrução (índice de Theil-T médio $=0,302$ ), abrange níveis de escolaridade bem distintos. Há indícios de que essas diferenças com relação à escolaridade podem estar, também, ligadas às leis que regulamentam o mercado de trabalho local e aos pisos salariais de profissões.

No que se refere ao gênero, a desigualdade mantém-se relativamente elevada ao longo do tempo, mostrando-se maior entre homens (índice de Theil-T $=0,375$ ) do que entre mulheres (índice de Theil-T =0,365).

Entre as categorias das variáveis estruturais do mercado de trabalho, as desigualdades por natureza jurídica dos estabelecimentos têm demonstrado uma propensão à redução. A esfera de atuação privada foi a que apresentou a maior redução. $O$ coeficiente de Theil-T que, em 2000 , era de 0,324 , caiu, em 2009 , para 0,228 , ou seja, houve uma redução de $42 \%$. Na esfera pública, esse decréscimo ficou em, aproximadamente, $31 \%$. 
Da mesma forma, as desigualdades nas categorias das atividades produtivas mostram uma perspectiva de redução. Todavia, sobressaem-se os serviços em arrolamento à má distribuição de renda (índice de Theil-T médio $=0,341$ ). Além disso, o índice de Theil-T para a construção civil e a agropecuária parece não refletir bem a realidade distributiva da remuneração nesses setores. Provavelmente, isso seja consequência da grande informalidade que permeia essas atividades econômicas.

Conforme Bêrni, Marquetti e Kloeckner (2002), o eixo agricultura-serviços constitui o círculo vicioso entre êxodo rural e informalização urbana da economia. Esses dois fenômenos perpetuam as desigualdades, constituindo empecilhos ao surgimento de uma sociedade mais igualitária.

\section{CONCLUSÃO}

O índice de Theil consiste em uma medida de desigualdade bastante útil por duas razões: em primeiro lugar, por permitir decompor as disparidades em determinantes como gênero, classe social, faixa etária, escolaridade, setores de atividade, por exemplo; e, em segundo lugar, por possibilitar uma análise microrregional, em concordância com a realidade local, considerando as características específicas associadas ao lugar.

No estudo de caso contemplado neste artigo, referente ao mercado de trabalho formal de Santa Maria, verificou-se que a variável faixa etária apresentou os índices de desigualdade mais elevados, seguida pelo gênero dos trabalhadores e pela atividade econômica em que estes se encontravam inseridos. Diante disso, sugere-se, para trabalhos futuros, que o índice de Theil-T seja aplicado para determinantes das desigualdades que relacionem os investimentos em capital humano, o melhor encadeamento entre atividades produtivas, as características pessoais como a experiência profissional relacionada à idade e a regulamentação do mercado de trabalho.

\section{REFERÊNCIAS}

ARAUJO, J. R.; SALVATO, M. A.; SOUZA, P. F. L. Decomposição do índice de Theil-T em disparidades regionais, entre gêneros, raciais e educacionais: uma análise da desigualdade de renda da Região Sul. In: ENCONTRO REGIONAL DE ECONOMIA - ANPEC - SUL, 9., 2008, Curitiba. Anais... Curitiba: 2008. p. 1-20.

AREND, M. Origens e determinantes dos desequilíbrios no Rio Grande do Sul: uma análise a partir da Teoria Institucional de Douglass North. Ensaios FEE, v. 26, n. especial, p. 63-94, 2005.

BÊRNI, D. A; MARQUETTI, A; KLOECKNER, R. A desigualdade Econômica no Rio Grande do Sul: primeiras investigações sobre a curva de Kuznets. Ensaios FEE, v. 23, n. especial, p. 443466, 2002.

BAISCH, L. da V. et al. Gestão estratégica e as
Competências gerenciais no departamento de marketing: estudo de uma indústria de bebidas. Revista de Administração da UFSM, Santa Maria, v. 5, n. especial, p. 729-748, dez. 2012.

DENARDIN, E. S. et al. Os tipos de inovações implementadas nos empreendimentos de economia solidária do projeto Esperança/ Cooesperança de Santa Maria - RS. Revista de Administração da UFSM, Santa Maria, v. 5, n. especial, p. 651-666, dez. 2012.

FIGUEIREDO, E. A.; ZIEGELMANN, F. A. Mudança na distribuição de renda brasileira: significância estatística e bem-estar econômico. Economia Aplicada, v. 13, n. 2, p. 257-277, 2009.

FROSINI, B. V. Aproximation and decomposition of Gini, Pietra-Ricci and Theil inequality measures. Empirical Economics, n. 43, p. 175197, 2012.

FUNDAÇÃO JOÃO PINHEIRO. Definição e 
Metodologia de Cálculos dos Indicadores e Índices de Desenvolvimento Humano e Condições de Vida, 1998. Disponível em: http:// www.conei.sp.gov.br/ind/MetodologiasIDHMelCV.pdf. Acesso em: 25 nov. 2013.

HOFFMANN, R. Estimação da desigualdade dentro de estratos no cálculo do índice de Gini e da redundância. Pesquisa e Planejamento Econômico, v. 3, n. 9, dez. 1979.

. Distribuição de renda: medidas de desigualdade e pobreza. São Paulo: Edusp, 1998.

. Estatística para economistas. 4. ed. São Paulo: Pioneira Thomson Learning, 2006.

MATOS, J. D. de. Distribuição de renda: fatores condicionantes e comparação entre as regiões metropolitanas pesquisadas pela PED. Porto Alegre: FEE, 2005.

LENGLER, L. Uma análise do comportamento empreendedor e do processo decisional de presidentes de associações apícolas no Rio Grande do Sul. Revista de Administração da UFSM, Santa Maria, v. 1, n. 1, p. 153-170, jan./ abr. 2008.

RAMOS, L. Interpretando variações nos índices de desigualdade de Theil. Pesquisa e Planejamento Econômico, v. 20, n. 3, p. 479488, dez. 1990.

A desigualdade de rendimentos do trabalho no período pós-real: o papel da escolaridade e do desemprego. Economia Aplicada, São Paulo, v. 11, n. 2, p. 281-301, abr./jun. 2007.

RAMOS, L.; VIEIRA, M. L. Desigualdades de rendimentos no Brasil nas décadas de 80 e 90: evolução e principais determinantes. Textos para discussão, n. 803, jun. 2001.

SANTOS, M. P. dos. Distribuição de renda e desenvolvimento humano: estado de São Paulo 1970-2000. São Paulo: Educ, 2007.
SHANNON, C. E. A mathematical theory of communication. The Bell System Technical Journal. v. 27, p. 379-423, 623-656, Jul./Oct. 1948. 